\title{
Evaluation Of The Relationship Between Affective Temperament and Depression at Postpartum Period
}

\author{
İbrahim Eren Pek ${ }^{1}$, Halil İbrahim Taş², Kürşat Altınbaş ${ }^{3}$ \\ ${ }^{1}$ Department of Obstetrics and Gynecology, Dinar State Hospital, Afyon/TURKEY, ${ }^{2}$ Department of Psychiatry, Çanakkale Onsekiz Mart \\ University, Çanakkale/TURKEY, ${ }^{3}$ Department of Psychiatry, Selçuk University, Konya/TURKEY
}

\section{Objective}

Risk of mood episodes, especially depression, is higher at postpartum period. Considering the data indicating that affective temperamental features are subclinical forms of mood disorders, we aimed to evalute the prevalence of depression and its relationship between affective temperament at post partum period.

\section{Methods}

The study included 164 postpartum women. The participants were administered Edinburgh Postnatal Depression Scale (EPDS), modified Hypomania Checklist (mHCL-32) and Temperament Evaluation of Memphis, Pisa, Paris, and San Diego Autoquestionnaire (TEMPS-A) scales.

Table 1. Descriptives of Rating Scale Scores

\begin{tabular}{|l|c|c|c|c|c|}
\hline & Min. & Max. & Mean & Med. & SD \\
\hline EPDS & 4.0 & 18.0 & 7.5 & 7.0 & 2.5 \\
\hline mHCL-32 & 0.0 & 11.0 & 4.4 & 4.0 & 2.4 \\
\hline $\begin{array}{l}\text { TEMPS-A } \\
\text { depressive }\end{array}$ & 3.0 & 13.0 & 6.8 & 7.0 & 1.9 \\
\hline $\begin{array}{l}\text { TEMPS-A } \\
\text { cyclothymic }\end{array}$ & 3.0 & 8.0 & 5.4 & 5.0 & 1.2 \\
\hline $\begin{array}{l}\text { TEMPS-A } \\
\text { hyperthymic }\end{array}$ & 2.0 & 10.0 & 5.6 & 5.0 & 1.9 \\
\hline $\begin{array}{l}\text { TEMPS-A } \\
\text { irritable }\end{array}$ & 1.0 & 9.0 & 3.9 & 4.0 & 1.6 \\
\hline $\begin{array}{l}\text { TEMPS-A } \\
\text { anxious }\end{array}$ & 1.0 & 15.0 & 6.0 & 5.5 & 2.7 \\
\hline EPDS: Edinburg & & & & & \\
\hline
\end{tabular}

EPDS: Edinburgh Postnatal Depression Scale, $\boldsymbol{m H C L - 3 2 : ~ M o d i f i e d ~}$ Hypomania Check List, TEMPS-A: Temperament Evaluation of Memphis, Pisa, Paris, and San Diego Autoquestionnaire Scale. Min:Minimum, Max:Maximum, Med:Median, SD:Standard Deviation

\section{Results}

Mean age of the women was $23.8 \pm 2.9$ years while mean age of their partners was $25.8 \pm 3.1$ years. Most of the women reported that their pregnancy was not planned(76.8\%, $\mathrm{n}=126)$. The prevalence of depression according to the EPDS cut-off score was $11 \%(n=18)$. Depression scores were positively correlated with irritable temperament scores $(\mathrm{rho}=0.16, \mathrm{p}=0.04)$ and mHCL-32 scores $(\mathrm{rho}=0.2, \mathrm{p}=0.01)$. There was no statistically significant difference between the women with lower and higher EPDS scores in terms of previous pregnancy and abortus history,

Table 2. Correlation Analysis of Depression and Temperament Scores

\begin{tabular}{|c|c|c|c|c|c|}
\hline & $\begin{array}{c}\text { Depressive } \\
\text { Temp. }\end{array}$ & $\begin{array}{c}\text { Cyclothy mic } \\
\text { Temp. }\end{array}$ & $\begin{array}{c}\text { Hyperthymic } \\
\text { Temp. }\end{array}$ & $\begin{array}{c}\text { Irritable } \\
\text { Temp. }\end{array}$ & $\begin{array}{c}\text { Anxious } \\
\text { Temp. }\end{array}$ \\
\hline $\begin{array}{c}\text { EPDS } \\
\text { rho } \\
\boldsymbol{p}\end{array}$ & 0.17 & 0.07 & -0.09 & 0.16 & 010 \\
\hline $\mathbf{m H C L - 3 2}$ & 0.11 & 0.39 & 0.24 & $0.04 *$ & 0.22 \\
\hline $\boldsymbol{r h o}$ & 0.10 & -0.08 & -0.11 & 0.10 & 0.10 \\
\hline $\boldsymbol{p}$ & 0.22 & 0.29 & 0.17 & 0.89 & 0.18 \\
\hline
\end{tabular}

EPDS: Edinburgh Postnatal Depression Scale, mHCL-32: Modified Hypomania Check List, Temp: Temperament, ${ }^{*} p<0.05$ is statisticallysignificant

\section{Conclusion}

Depression should be closely screened during postparum period. Irritable temperament might be an indicator of depression for women after child birth. Prospective longterm follow up studies with big sample size are required to unveil the clinical characteristics of postpartum depression

1.Çelik SB, Bucaktepe GE, Uludag A, Bulut IU, Erdem Ö, Altinbas K.Screening mixed depression and bipolarity in the postpartum period at a primary health care center. Compr Psychiatry. 2016 Nov; 71:57-62.

2. Sherman BJ, Vousoura E, Wickramaratne P, Warner V, Verdeli H.Temperament and major depression: How does difficult temperament affect frequency, severity, and duration of major depressive episodes among offspring of parents with or without depression?mJourn Affect Disord. 2016 Aug:200:82-8.

3.Gurpegui D, Ortuño F, Gupegui M. Temperament traits and remission of depression: A naturalistic six-month longitudinal study. J Affect Disord. 2019 Jan 15;243:494-502. 